\title{
Monitoring the New Circular Pedestrian Steel Bridge over the São Roque and Botirões Channels with Weldable Fiber-Bragg Grating Sensors
}

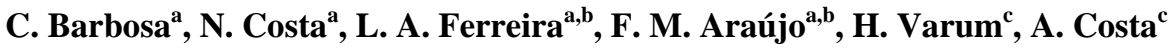 \\ ${ }^{a}$ FiberSensing, R. Vasconcelos Costa, 277, 4470-640 Maia, Portugal \\ ${ }^{b}$ INESC Porto, Campus da FEUP, R. Dr. Roberto Frias, 378, 4200-465 Porto, Portugal \\ ${ }^{c}$ Secção Autónoma de Engenharia Civil, Universidade de Aveiro, Campus Universitário de Santiago, 3810-193 Aveiro, Portugal \\ Corresponding author: L. A. Ferreira, luis.ferreira@fibersensing.com
}

\begin{abstract}
To monitor a new circular pedestrian bridge, new strain and temperature sensors based on fiber Bragg gratings were developed to be directly welded to the steel structure, as well as proper protection and installation procedures.

OCIS codes: $060.2370,060.2340$.
\end{abstract}

\section{Introduction}

In the last few years, the examples of application of fiber Bragg grating sensors for structural health monitoring have become more and more frequent [1,2]. The explanation for this fact relies not only on the particularly appropriate characteristics associated to these devices for measuring the most relevant structural parameters, without revealing a number of disadvantages usually attributed to conventional sensors, but also on the growing number of companies operating in the area of fiber optic sensing [3]. Even if standardization still seems a little bit far for fiber Bragg grating based sensing systems (up to now, only a few isolated efforts are known in this direction [4,5]), these companies are already offering nowadays an interesting range of sensors based on this technology, as well as several types of measurement units with increasing capabilities. From the point of view of the sensors, important evolutions have been made in terms of parameters that can be measured, packaging oriented to a variety of applications, and long-term reliability. In what concerns the measurement units, new improvements included increasing the number of interrogated sensors, higher dynamic ranges and sampling rates, more compactness and portability, and inclusion of data logging and data transmission capabilities [6].

Nevertheless, real world applications are always fruitful in terms of new challenges. If the fiber Bragg grating based sensors are intended to monitor real structures, they must be specifically tailored to fulfill the requirements of existing applications and also, most important, must be comprehensible to the technicians that are familiar with conventional electric based instrumentation.

One interesting case is the monitoring of large steel structures such as bridges. For this particular application, fiber Bragg grating sensors should follow the most suitable product design available from the conventional instrumentation world, which is the weldable strain gage. This type of sensor is clearly preferable since it minimizes surface preparation requirements and precludes epoxy curing processes during installation. Instead, spot welding is used, which can be accomplished with a portable stored-energy hand-probe spot welder. This helps reducing the installation time and also ensures proper performance of the sensor at higher temperatures and harsh-environments, rendering unnecessary the use of elevated-temperature-curing adhesives. On the other hand, environmental protection can be as easily applied to a welded sensor as to an adhesively bonded gage.

For monitoring the new circular pedestrian steel bridge over the São Roque and Botirões channels in Aveiro, Portugal (Figure 1 and Figure 2), new strain sensors based on fiber Bragg gratings were developed for being directly welded to the steel structure following similar procedures to those of conventional weldable strain gages. For referencing proposes, weldable fiber-Bragg grating based temperature sensors were also developed. In addition, proper protection means and installation procedures were established and implemented. Testing results on sensors reveal linear response for the required measuring range and stability under cyclic loading and temperature ageing.

The bridge instrumentation followed a monitoring plan designed accordingly to previous numerical analysis of the structure. The measurements obtained with the installed fiber Bragg grating sensing network will allow the improvement and calibration of the numerical models, as well as the real-time monitoring of the bridge structural health during loading tests and service. 


\section{Weldable sensors}

Figure 3 shows the developed weldable fiber Bragg grating strain gage. It consists of a capillary stainless steel tube containing the sensing element, which is laser welded to a thin stainless steel base that is used for direct spot welding to the steel structure. The input/output fibers are protected with standard $900 \mu \mathrm{m}$ buffer. For installation purposes, a protective stainless steel cap was also developed that can be directly welded to the structure by using the same hand-probe spot welder (Figure 4). Input/output fibers enter/exit this cap already protected by $3 \mathrm{~mm}$ pvc buffer with internal stainless steel coil. The weldable temperature sensor has exactly the same mechanical design as the strain one. The only difference is that the sensing element inside the capillary stainless steel tube is properly strain isolated.

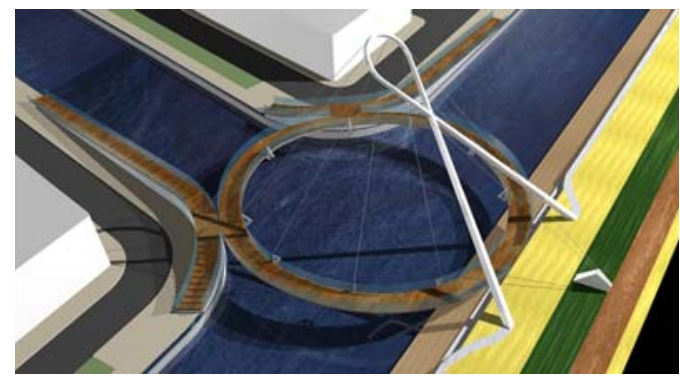

Figure 1. Project of the new circular pedestrian steel bridge over the São Roque and Botirões channels in Aveiro, Portugal.

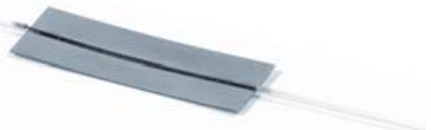

Figure 3. Fiber Bragg grating weldable strain (or temperature) sensor.

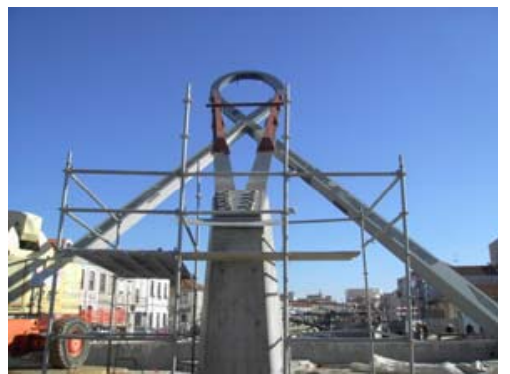

Figure 2. Construction of the new circular pedestrian steel bridge over the São Roque and Botirões channels in Aveiro, Portugal.

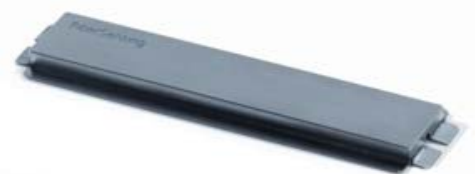

Figure 4. Protection cap for fiber Bragg grating weldable sensors.

To evaluate the performance of the strain weldable sensor, it was first spot welded to a stainless steel bar, as shown in Figure 5, and then submitted to a load test in a standard mechanical testing machine (Figure 6). For calibration, the Bragg wavelength was monitored while increasing and decreasing load was applied to the bar at constant temperature. The sensor response is shown in Figure 7, which reveals a sensitivity of $1.2 \mathrm{pm} / \mu \varepsilon$. The bar was then submitted to thousands of load cycles in order to evaluate the stability of its response under repeated loading. As can be checked from the results in Figure 8, the sensor response is stable and reliable.

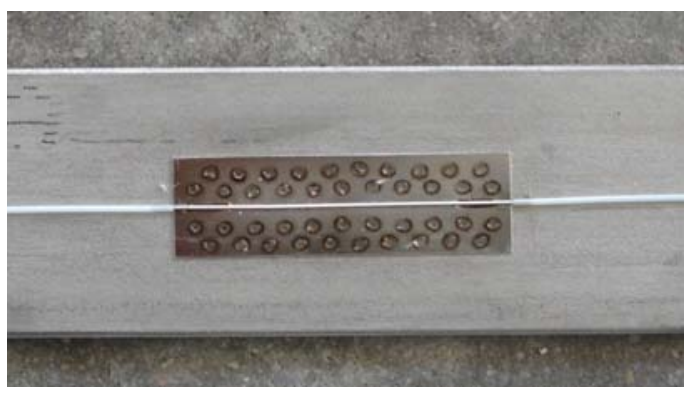

Figure 5. Strain sensor welded to a stainless steel bar.

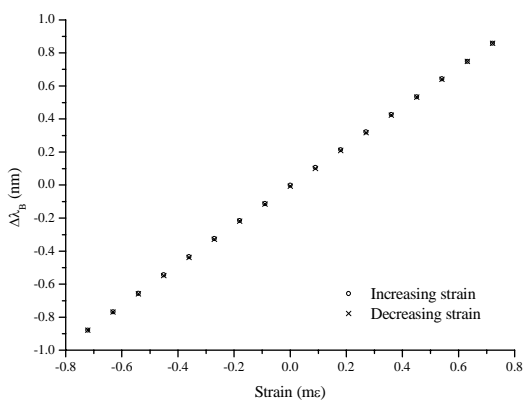

Figure 7. Calibration of the weldable strain sensor.
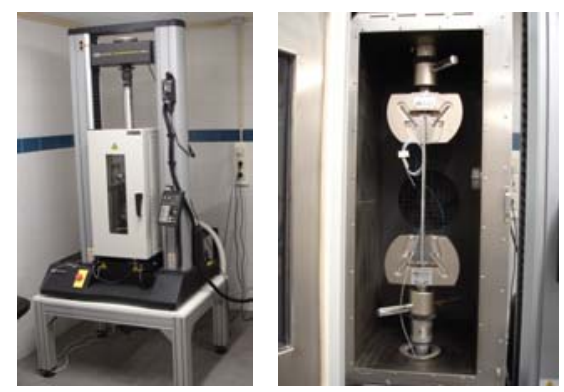

Figure 6. Instrumented bar in the mechanical testing machine.

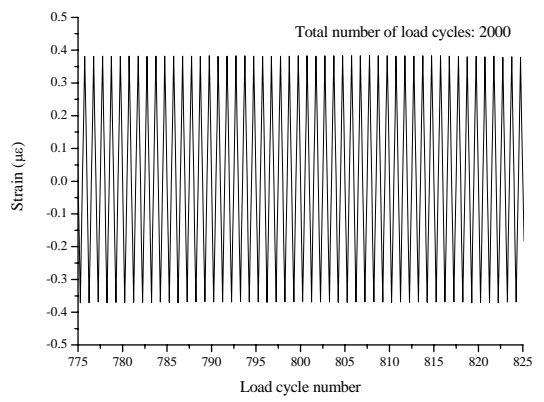

Figure 8. Weldable strain sensor response under cyclic loading. 
To characterize the weldable temperature sensor, it was placed in a climatic chamber and the Bragg wavelength was monitored while the temperature was varied. Figure 9 shows the obtained result for a single temperature cycle. From this result, a linear sensitivity of $10 \mathrm{pm} /{ }^{\circ} \mathrm{C}$ can be estimated for the expected environmental temperature range. The long-term reliability of the sensor was also evaluated applying a few consecutive high temperature cycles. Data in Figure 10 indicate no significant degradation of the sensor response with subsequent temperature ageing.

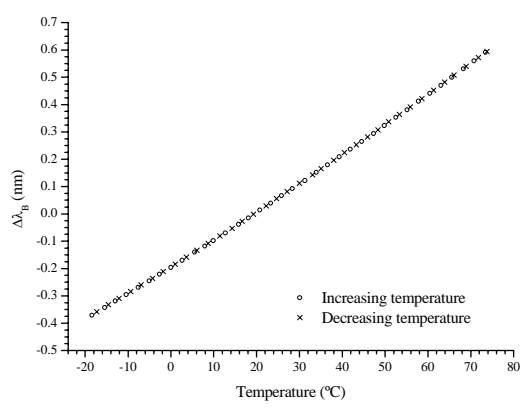

Figure 9. Calibration of the weldable temperature sensor.

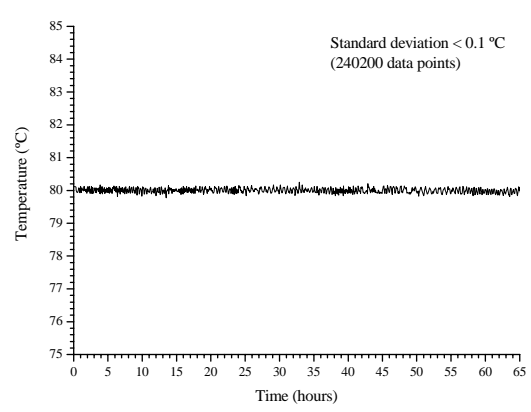

Figure 10. Weldable temperature sensor ageing.

\section{Sensing network}

The sensing network implemented in the circular pedestrian steel bridge under analysis is shown in Figure 11. It consists of 32 weldable strain sensors and 8 weldable temperature sensors deployed in a star configuration with 8 branches. Figure 12 shows a typical measurement branch. The access to all the sensor branches is concentrated in a connection box placed on the concrete block for the structure anchorage.

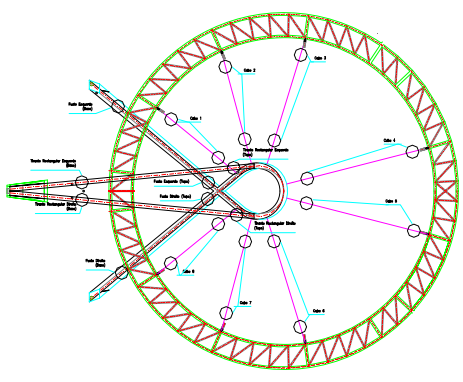

Figure 11. Bridge monitoring plan.

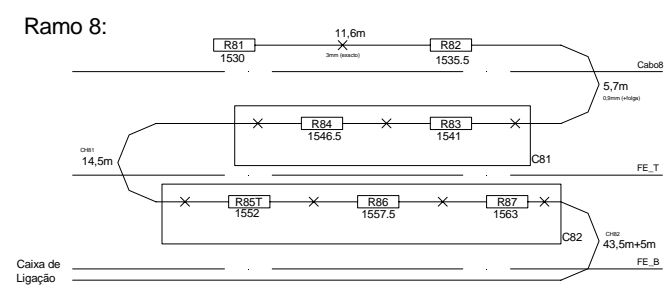

Figure 12. A typical branch of the network.

The monitoring plan was drawn accordingly to previous numerical analysis of the structure. The configuration of the sensing network was further determined by aesthetic considerations, since it was important to minimize any visual impact in the structure. It is expected the fiber optic strain sensing network to give the stress distribution in the most relevant structural elements of the bridge, namely the suspension cables and beams. The measurements obtained with the installed fiber Bragg grating sensing network will allow the improvement and calibration of the predicted models, as well as the real-time monitoring of the bridge structural health during loading tests and service. The temperature sensing network was defined accordingly to the localization of the strain sensors and considering regions where temperature is assumed identical.
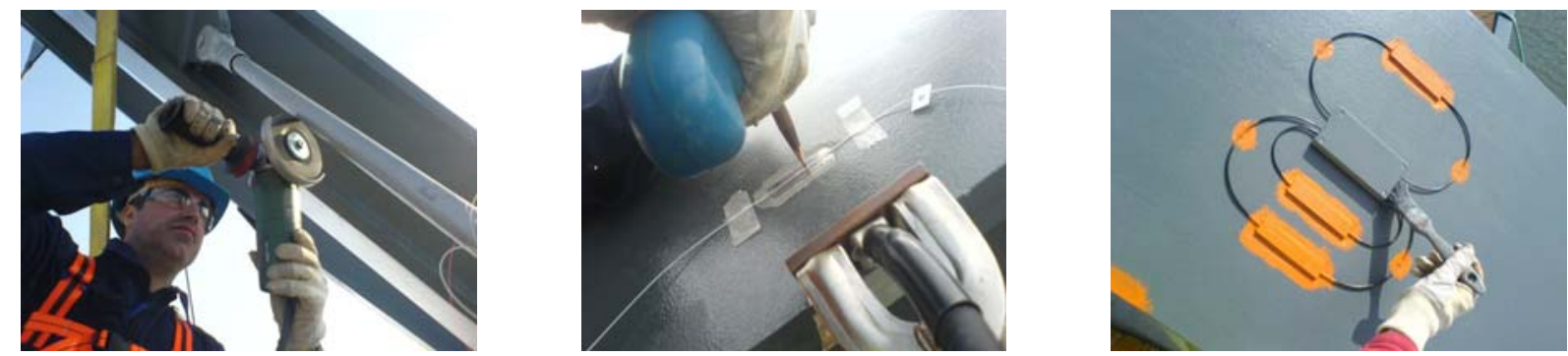

Figure 13. Installation process of a weldable sensor: regularization and cleaning of the surface, sensor welding, and sensor protection.

The installation of the sensing network started after the bridge structure was already constructed. This fact has increased the difficulties associated with the deployment of the sensors and connection cables. Therefore, special care was taken to make easier the installation processes while ensuring proper protection of all the sensors and connections 
to the environment action, particularly in what concerns moisture. The installation of each sensor comprised three phases: regularization and cleaning of the surface, welding of the sensor, and protection of the welded sensor. After the welding process, all the sensors were covered with hermetic tape before being protected with the weldable cap, which was sealed afterwards. Figure 13 exemplifies the complete process of installation of a sensor in the bridge. Even if this process is not complex, installation of the network in the constructed bridge was not an easy task, especially in what concerns the cylindrical suspension cables, as can be understood from Figure 14. All the fibers in the network ( $\sim 5 \mathrm{~km}$ in total) were protected with $3 \mathrm{~mm}$ pvc buffer with internal stainless steel coil, and fixed to the steel structure by using weldable straps - Figure 15. Connections and splices in the network were accommodated in small hermetic boxes that were also spot welded to the structure, also shown in Figure 15. The performance of all the sensors during and after the installation process was monitored using a portable battery powered measurement unit from FiberSensing (Portable BraggMeter [7]), which is shown in Figure 16. As an example, the spectra of the sensors corresponding to branch 8 are shown in Figure 17, obtained with the BraggMeter after installation.

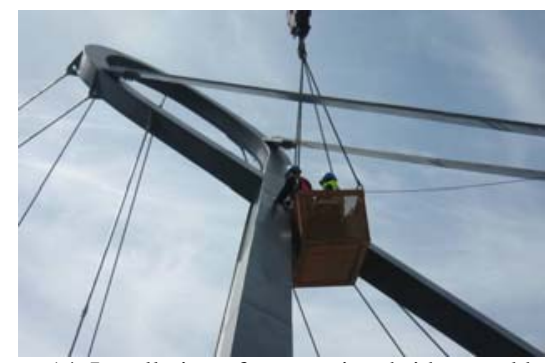

Figure 14. Installation of sensors in a bridge steel beam.

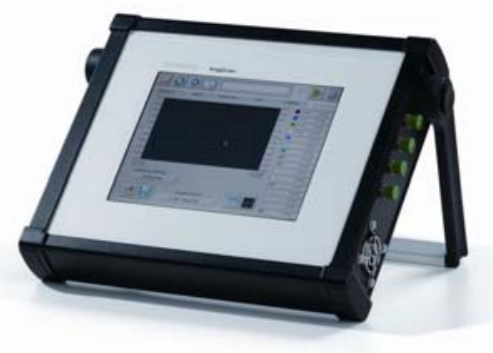

Figure 16. Portable BraggMeter.

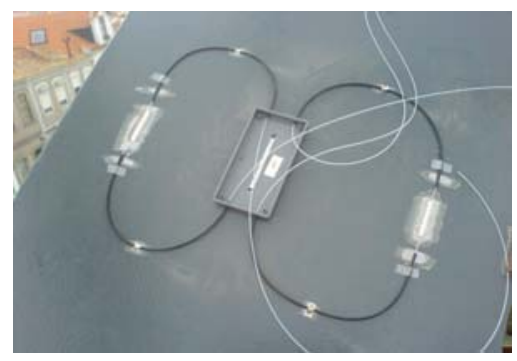

Figure 15. Boxes for accommodation of connections and splices.

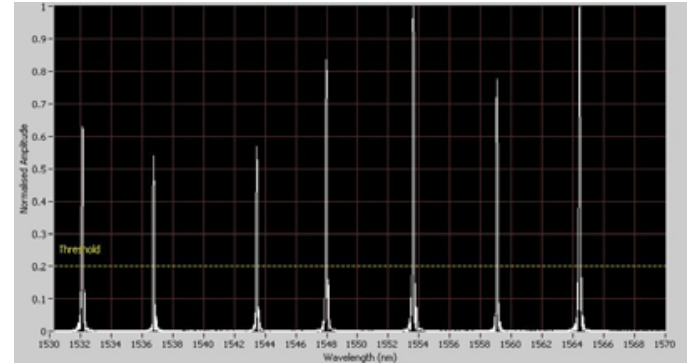

Figure 17. Reflected spectra from sensing branch 8.

\section{Final comments}

New temperature and strain fiber Bragg grating sensors were developed for installation in a new circular pedestrian steel bridge over the São Roque and Botirões channels in Aveiro, Portugal. The sensors were developed for being directly welded to the steel structure following similar procedures to those of conventional weldable strain gages. In addition, proper protection means and installation procedures were established and implemented. The obtained results evidence linear response of the sensors for the required measuring range, and stability over load and temperature cycles. The deployment of the sensing network on the bridge also revealed to be practical albeit the particularly difficult installation conditions. The implemented fiber Bragg grating sensing network will be used in the near future for load tests, and will also allow the real-time monitoring of the bridge structural health during service.

Acknowledgements: the authors acknowledge the bridge designers, Luís Viegas and Domingos Moreira, and AVEIROPOLIS.

\section{References}

[1] K. Kincade, "Fiber sensors lay groundwork for structural health monitoring”, Laser Focus World 42 (2), $63-67$ (2005).

[2] Sensing issues in civil structural health monitoring, F. Ansari, ed. (Springer, Dordrecht, 2005).

[3] S. D. Crossley, "The commercialization of fibre optic sensors", in Handbook of optical fibre sensing technology, J. M. López-Higuera, ed. (John Wiley \& Sons, Chichester, 2002).

[4] E. Riviera, A. A. Mufti, D. J. Thomson, "Civionics specifications for fiber optic sensors for structural health monitoring”, in Proceedings of the Second International Workshop on Structural Health Monitoring of Innovative Civil Engineering Structures, A. Mufti, F. Ansari, eds. (ISIS Canada Corporation, Winnipeg, 2004).

[5] E. Udd, D. Inaudi, B. Culshaw, W. Ecke, "Fiber optic sensor opportunities and obstacles for aerospace and civil structure applications", Panel Discussion in Smart Sensor Technology and Measurement Systems - SPIE Conference 5384 (Smart Structures/NDE 2004, San Diego, 2004).

[6] T. Graver, D. Inaudi, J. Doornink, “Growing market acceptance for fiber-optic solutions in civil structures”, in Proceedings of Fiber Optic Sensor Technology and Applications III, SPIE Vol. 5589, M. A. Marcus, B. Culshaw, J. P. Dakin, eds., 44-55 (2004).

[7] Portable BraggMeter Data Sheet, available in www.fibersensing.com. 